\begin{tabular}{|c|c|}
\hline Title & Bulk Energy Dissipation Mechanism for the Fracture of Tough and Self-Healing Hydrogels \\
\hline Author(s) & $\begin{array}{l}\text { Sun, T ao Lin; Luo, Feng; Hong, Wei; Cui, Kunpeng; Huang, Y iwan; Zhang, Hui Jie; King, Daniel R.; Kurokawa, } \\
\text { T akayuki; Nakajima, T asuku; Gong, Jian Ping }\end{array}$ \\
\hline Citation & $\begin{array}{l}\text { Macromolecules, 50(7), 2923-2931 } \\
\text { https://doi.org/10.1021/acs.macromol.7b00162 }\end{array}$ \\
\hline Issue Date & $2017-04-11$ \\
\hline Doc URL & http:/hdl.handle.net/2115/68615 \\
\hline Rights & $\begin{array}{l}\text { This document is the A ccepted Manuscript version of a Published Work that appeared in final form in Macromolecules, } \\
\text { copyright @2017 A merican Chemical Society after peer review and technical editing by the publisher. To access the } \\
\text { final edited and published work see http://pubs.acs.org/doi/abs/10.1021/acs.macromol.7b00162 }\end{array}$ \\
\hline Tyре & article (author version) \\
\hline Additional Information & There are other files related to this item in HUSCAP. Check the above URL. \\
\hline File Information & Macromol50- 7 2923-2931.pdf \\
\hline
\end{tabular}

Instructions for use 
This document is confidential and is proprietary to the American Chemical Society and its authors. Do not copy or disclose without written permission. If you have received this item in error, notify the sender and delete all copies.

\section{Bulk Energy Dissipation Mechanism for the Fracture of Tough and Self-Healing Hydrogels}

\begin{tabular}{|r|l|}
\hline Journal: & Macromolecules \\
\hline Manuscript ID & ma-2017-001623.R1 \\
\hline Manuscript Type: & Article \\
\hline Complete List of Authors: & $\begin{array}{l}\text { Sun, Tao Lin; Hokkaido University, Graduate School of Science } \\
\text { Luo, Feng; College of Polymer Science and Engineering, } \\
\text { Hong, Wei; Iowa State University, Aerospace Engineering } \\
\text { Cui, Kunpeng; Hokkaido University, Laboratory of Soft and Wet Matter } \\
\text { Huang, Yiwan; Hokkaido University, Department of Transdisciplinary Life } \\
\text { Science } \\
\text { Zhang, Hui Jie; Hokkaido University } \\
\text { King, Daniel; Hokkaido University, Faculty of Advanced Life Science } \\
\text { Kurokawa, Takayuki; Hokkaido University } \\
\text { Nakajima, Tasuku; Hokkaido University, Faculty of Advanced Life Science } \\
\text { Gong, Jian Ping; Hokkaido University, Graduate School of Science }\end{array}$ \\
\hline
\end{tabular}




\title{
Bulk Energy Dissipation Mechanism for the Fracture of Tough and Self-Healing Hydrogels
}

\author{
Tao Lin Sun ${ }^{1,2 *}$, Feng Luo ${ }^{1}$, Wei Hong ${ }^{2,3}$, Kunpeng Cui ${ }^{1}$, Yiwan Huang ${ }^{4}$, Hui Jie Zhang ${ }^{4}$, \\ Daniel R. King ${ }^{1,2}$, Takayuki Kurokawa ${ }^{1,2}$, Tasuku Nakajima ${ }^{1,2}$, Jian Ping Gong ${ }^{1,2 *}$ \\ ${ }^{1}$ Laboratory of Soft and Wet Matter, Faculty of Advanced Life Science, Hokkaido \\ University, Sapporo 001-0021, Japan \\ ${ }^{2}$ Global Station for Soft Matter, Global Institution for Collaborative Research and \\ Education (GI-CoRE), Hokkaido University \\ ${ }^{3}$ Department of Aerospace Engineering, Iowa State University of Science and \\ Technology, Ames, IA 50010, USA \\ ${ }^{4}$ Laboratory of Soft and Wet Matter, Graduate School of Life Science, Hokkaido \\ University, Sapporo 001-0021, Japan
}

Key Words: Polyampholyte hydrogel; Viscoelasticity; Fracture behavior; Self-healing; Ionic bond; Time-temperature superposition

\begin{abstract}
Recently, many tough and self-healing hydrogels have been developed based on physical bonds as reversible sacrificial bonds. As breaking and reforming of physical bonds are time-dependent, these hydrogels are viscoelastic and the deformation rate and temperature pronouncedly influence their fracture behavior. Using a polyampholyte hydrogel as a model system, we observed that the time-temperature superposition principle is obeyed not only for the small strain rheology, but also for the large strain
\end{abstract}


hysteresis energy dissipation and the fracture energy below a certain temperature. The three processes possess the same shift factors that obey the equation of Williams, Landel, and Ferry (WLF) time-temperature equivalence. The fracture energy $\Gamma$ scales with the crack velocity $V_{c}$ over a wide velocity range as, $\Gamma \sim V_{c}^{\alpha}(\alpha=0.21)$. The exponent $\alpha$ of the power law is well-related to the exponent $\kappa$ of the relaxation modulus $G(\mathrm{t}) \sim t^{-\kappa} \quad(\kappa=0.26)$, obeying the prediction $\alpha=\kappa /(1+\kappa)$ from classic viscoelasticity theory. These results show that the fracture energy of the polyampholyte gel is dominated by the bulk viscoelastic energy dissipated around the crack tip. This investigation gives an insight into designing tough and self-healing hydrogels and predicting their fracture behaviors from their dynamic mechanical spectrum. 


\section{INTRODUCTION}

Over the past decade, various mechanically strong and tough hydrogels have been invented, which substantially broaden the possible uses of hydrogels in hygiene and medical fields, for example, as cell scaffolds, drug delivery agents, and load bearing structural biomaterials. ${ }^{1-12}$ The basic principle for developing tough hydrogels is to incorporate energy dissipation structures (sacrificial bonds) into the hydrogels. Generally, these tough gels can be classified into two categories depending on their molecular mechanisms to dissipate energy. One is elastic double network hydrogels. ${ }^{2,3}$ The energy dissipation of these hydrogels is by the breaking of sacrificial covalent bonds of the brittle network, which is independent of the observation time window in the conventional time scale. The other is viscoelastic supramolecular hydrogels. ${ }^{6,10,13}$ These hydrogels contain abundant physical bonds and energy is dissipated by breaking these sacrificial physical bonds, which strongly depends on the observation time window. Nano-composite hydrogels, such as those with clay or silica nano-particles, also belong to the latter type whereupon the energy dissipation is believed to occur mainly at the polymer-inorganic composite interface. ${ }^{11,12,14}$ The viscoelastic tough hydrogels usually exhibit self-resilient and self-healing behaviors due to the reversible nature of physical bonds. ${ }^{9,13,15}$

For the application as load-bearing materials, understanding the failure mechanism and predicting the failure behavior of these tough hydrogels are indispensable. In the case of the elastic double network hydrogels, it has been shown that the energy required to advance a crack is dissipated in the large damage zone formed in front of the crack tip, in which the brittle network is substantially damaged. ${ }^{16,17}$ Such a toughening mechanism has also been applied to double and triple network elastomer systems. ${ }^{18}$ In 
the case of viscoelastic supramolecular hydrogels, few are known. It is highly possible that the energy required to advance a fracture plane by one-unit area includes not only the energy necessary to break the polymer chains ahead of the crack tip, but also the bulk viscoelastic energy dissipated around the crack tip, in similar to viscoelastic solids, such as rubbers. ${ }^{19-28}$

The purpose of this work is to study the fracture behavior and its energy dissipation mechanism of tough supramolecular hydrogels with sacrificial physical bonds. As a model system, we use a polyampholyte (PA) physical hydrogel, which is strongly viscoelastic and exhibits excellent mechanical properties, such as high strength and extensibility, high fracture energy, self-resilience, and self-healing. ${ }^{10,13,29-33}$ The PA gel is synthesized from the radical copolymerization of cationic monomers and anionic monomers at a very high concentration with balanced charges. The polyampholytes thus obtained are topologically entangled and each polymer chain possesses oppositely charged ionic groups randomly distributed along the chain backbone. By dialysis of small counter-ions and co-ions in pure water, ionic associations between opposite charges on the polymer chains, both intra- and inter- chains, are switched on, which gives a micro-phase separated PA hydrogels containing $\sim 50 \quad w t \%$ of water. ${ }^{10,34-36}$ In our previous paper, the PA hydrogels were described by a dichotomic molecular picture of the elastic network with weak bonds and strong bonds, where upon the weak bonds can break to dissipate energy and reform to impart self-healing, and strong bonds can maintain the integrity of the physical hydrogel over much longer timescale. Given the micro phase separated structure and the wide distribution of relaxation time in the dynamic rheological spectrum, however, this dichotomic network picture is over simplified, as suggested by recent works on tough hydrogels with phase separation 
structures. ${ }^{34-36}$ In this work, we intend to use a more global viscoelastic picture to discuss the mechanical behaviors of the PA hydrogels.

The mechanical behaviors of the viscoelastic gels are strongly dependent on the relative relation of observation time and the physical bond life time. Furthermore, the life time of physical bonds depends not only on the bond association energy but also on the observation temperature, as the bond breaking probability is related to the ratio of bond energy to thermal energy. Thus, deformation rate and temperature pronouncedly influence the mechanical and fracture behavior of the physical PA gels, which is essentially different from double network hydrogels that contain only chemical crosslinkers.

In this work, three sets of experiments, the rheological test for small strain behavior, the cyclic tensile test for large strain behavior, and the tearing test for fracture behavior, were performed over a wide range of deformation rate and environmental temperature. The validity of Williams, Landel, and Ferry (WLF) time-temperature equivalence is discussed for the three sets of experimental results. The results are further analyzed using the classic viscoelasticity theory.

\section{EXPERIMENTAL}

Synthesis of hydrogels Tough PA hydrogels of stoichiometric charge composition were synthesized by the radical copolymerization of a cationic monomer 3-(methacryloylamino)propyl-trimethylammonium chloride (MPTC) and an anionic monomer $p$-styrenesulfonate $(\mathrm{NaSS})$ with a monomer ratio NaSS:MPTC $=0.525: 0.475$ at a total monomer concentration $2.1 \mathrm{M}$, as described in the previous work. ${ }^{10} \mathrm{~A}$ mixed aqueous monomer solution together with $0.25 \mathrm{~mol} \% \mathrm{UV}$ initiator, 2-oxoglutaric acid (relative to the total monomer concentration) was injected into a reaction cell consisting 
of a pair of glass plates with a 2-mm spacer, and the reaction cell was irradiated by UV light $(\sim 365 \mathrm{~nm})$ for 11 hours. After polymerization, the as-prepared gel was immersed in a large amount of water for one week to reach the equilibrium and to wash away the residual chemicals.

Rheological test Dynamic rheological test was performed with an ARES rheometer (advanced rheometric expansion system, Rheometric Scientific Inc.). The disk-shaped sample with a diameter of $15.0 \mathrm{~mm}$ and a thickness of $1.3 \mathrm{~mm}$ was fixed between metal plates by using super glue (Konishi Co., Ltd.). In order to prevent water evaporating from the gel, the sample was surrounded by water during the measurement. Prior to each measurement, the auto-strain function and manual gap adjustment were used to minimize compression on the sample. The rheological tests were performed over a wide range of frequency $(0.1 \mathrm{rad} / \mathrm{s} \sim 100 \mathrm{rad} / \mathrm{s})$ at different temperature environment $\left(0.1^{\circ} \mathrm{C}\right.$ $\sim 56{ }^{\circ} \mathrm{C}$ ) at a shear strain of $0.1 \%$. The temperature of water, controlled by a thermal bath, was increased step-wise from low to high. Before each measurement, the sample was held at the set temperature for $300 \mathrm{sec}$ to reach the equilibrium.

Cyclic tensile test The sample for the cyclic tensile test was cut into the dumbbell-shape with standard JIS-K6251-7 size (12.0 mm (gauge length) $\times 2.0 \mathrm{~mm}$ (width)). The sample thickness was $1.3 \mathrm{~mm}$. The test was performed on a tensile-compressive tester (Tensilon RTC-1310A, Orientec Co.) and the sample was stretched to a large engineering strain ( $\varepsilon$ $=2$ ) and unloaded to the original position with the same loading-unloading velocity in a water bath with different temperature $\left(8^{\circ} \mathrm{C}, 24^{\circ} \mathrm{C}, 40^{\circ} \mathrm{C}\right.$ and $\left.56^{\circ} \mathrm{C}\right)$. The nominal stress $\sigma$ was obtained from the tensile force divided by the initial cross-sectional area of sample. The strain rate, $\dot{\varepsilon}$, is defined as the ratio of stretch velocity to the gauge length $(12.0 \mathrm{~mm})$. Hysteresis or energy dissipation, $U_{h y s}$, is calculated from the area enclosed 
by the loading-unloading curve. Three measurements were performed for each sample and results are mean of the three trials.

Tearing test To measure the tearing energy of PA gels, the mode III tearing test, known as the trousers test, was performed on a commercial test machine (Tensilon RTC-1310A, Orientec Co). ${ }^{8,37}$ The sample was cut into a standard JIS-K6252 $1 / 2$ size $(50.0 \mathrm{~mm}$ length $\times 7.5 \mathrm{~mm}$ width) with an initial notch length $\sim 20.0 \mathrm{~mm}$. The two arms of the hydrogel were clamped tightly by metal plates to prevent the sample from slipping during the tearing test. To make sure the crack advanced along the central line through the entire sample, the two arms were set perpendicular to the object surface of the clamps. Then the lower clamp was stretched at a constant stretch velocity $V_{t}$ until the crack advanced through the entire sample, and the corresponding tearing force $F$ and stretched displacement $L$ were recorded during the test. To directly relate the local deformation to tearing energy at the crack tip during crack propagation, the crack propagation velocity $V_{c}$ was recorded in situ by a digital camera. To control the temperature and prevent water evaporating from the samples, the tearing test was performed in a water bath at a described temperature $\left(8{ }^{\circ} \mathrm{C}, 24{ }^{\circ} \mathrm{C}, 40^{\circ} \mathrm{C}\right.$ and $\left.56^{\circ} \mathrm{C}\right)$. The tearing energy $\Gamma$ was calculated using the relation

$$
\Gamma=2 F / w
$$

where $w$ is the thickness of the sample and $F$ is the constant tearing force at crack propagation. Three measurements were performed for each sample and results are mean of the three trials.

\section{RESULTS and DISCUSSION}


The polyampholyte physical gel, containing $52 w t \%$ water, is very tough. At a stretching strain rate of $0.14 \mathrm{~s}^{-1}$, it shows a clear yielding around strain 0.5 , a high fracture strength of $1.8 \mathrm{MPa}$, and a large fracture stain of 8 . At an angular frequency of $62.8 \mathrm{rad} / \mathrm{s}$, the hydrogel exhibits a softening temperature at $48.2{ }^{\circ} \mathrm{C}$. These results are consistent with the previous report. ${ }^{10}$ Previous reports also show that this gel possesses weak bonds with a relaxation time $\tau_{0} \sim 3 \mathrm{~s}$ and strong bonds with a relaxation time longer than $\sim 10^{5} \mathrm{~s}$, and a characteristic creep time longer than $10^{5} \mathrm{~s} .{ }^{10,13,31,32}$

Time-temperature superposition at small strain

a

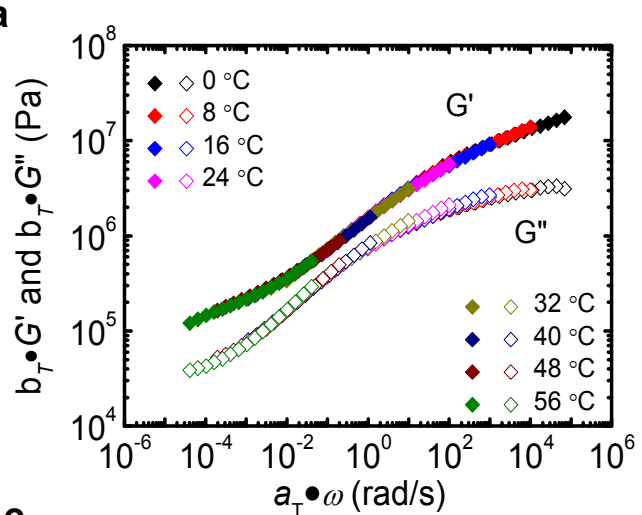

C

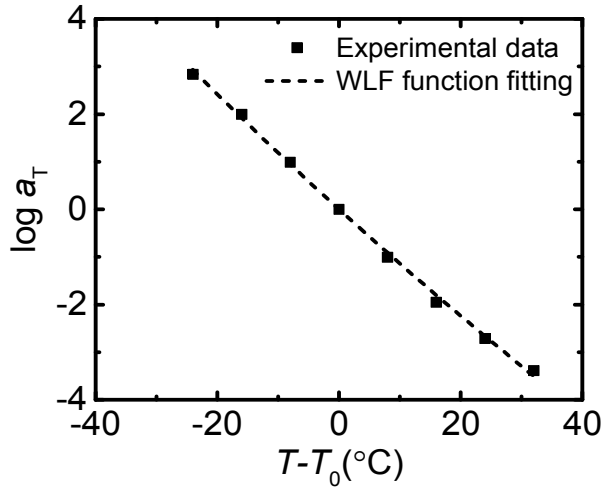

b

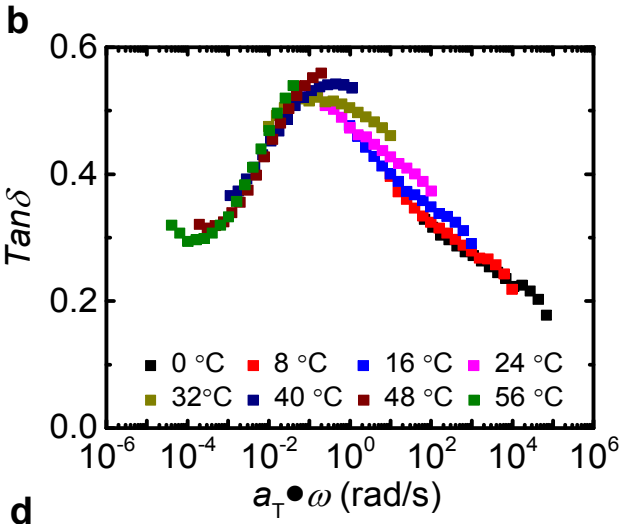

d

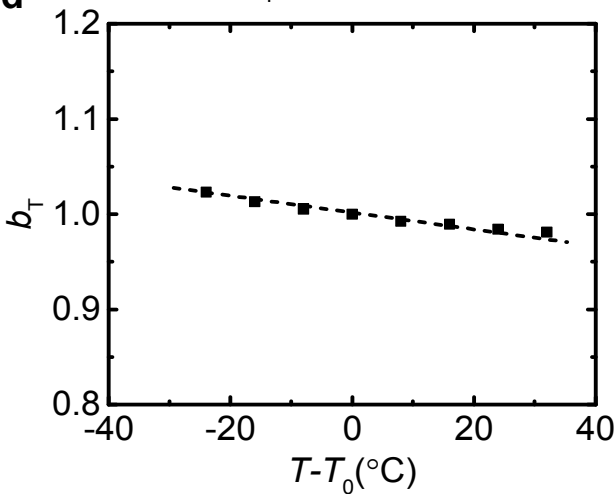

Figure 1 Linear dynamic behavior of polyampholyte (PA) hydrogels. (a, b) Constructed master curves of storage modulus $G^{\prime}$ and loss modulus $G^{\prime \prime}$ (a), and loss 
factor $\tan \delta$ (b) following the principle of time-temperature superposition. (c, d) Time-temperature horizontal shift factor $a_{T}$ (c) and vertical shift factor $b_{T}(\mathbf{d})$ as a function of temperature. Reference temperature $T_{0}=24{ }^{\circ} \mathrm{C}$.

According to the linear rheological theory, the principle of time-temperature superposition (TTS) holds for viscoelastic solids and it is possible to superimpose linear viscoelastic data taken at different temperature. The dynamic modulus at any given temperature, containing storage modulus $G^{\prime}$ and loss modulus $G^{\prime \prime}$, can be superimposed on data at a reference temperature $T_{0}$ using a time scale multiplicative horizontal shift factor $a_{T}$ and a modulus scale multiplicative vertical shift factor $b_{T}:{ }^{38}$

$$
\mathrm{G}^{\prime}(\mathrm{t}, \mathrm{T})=\mathrm{b}_{\mathrm{T}} \mathrm{G}^{\prime}\left(\frac{\mathrm{t}}{a_{T}}, \mathrm{~T}_{0}\right) \text { and } \mathrm{G}^{\prime \prime}(\mathrm{t}, \mathrm{T})=\mathrm{b}_{\mathrm{T}} \mathrm{G}^{\prime \prime}\left(\frac{\mathrm{t}}{a_{T}}, \mathrm{~T}_{0}\right)
$$

The $a_{T}$ and $b_{T}$ are treated as adjustable parameters, related to the temperature dependence of diffusion coefficient and variations in density, respectively. We first performed the linear viscoelastic test by frequency sweep at different temperatures (Figure S1). According to the TTS principle, we construct master curves of $G^{\prime}, G^{\prime \prime}$ and $\tan \delta$ for the polyampholyte hydrogel at a reference temperature of $24^{\circ} \mathrm{C}$. As shown in Figure 1a and b, master curves can be constructed very well over a wide frequency range of $10^{-5} \sim 10^{5} \mathrm{rad} / \mathrm{s}$. To further check the validity of time-temperature superposition, phase angle $\delta$ versus the complex modulus $G^{*}$ was plotted in Figure S1. This way of plotting can yield the temperature-independent curves when time-temperature superposition holds. Results exhibit a smooth curve of $\delta$ vs $G^{*}$ curve in the range of performed temperature, indicating that the ionic bonds, regardless of their wide distribution in strength, have the same temperature dependence and the physical PA gel shows this thermorheologically simple behavior. ${ }^{39,40}$ We also found that, 
at high temperature (from $56{ }^{\circ} \mathrm{C}$ to $95{ }^{\circ} \mathrm{C}$ ), the TTS does not hold, indicating that some structure changes occur at elevated temperature although the gel still looks opaque (data are not shown).

We further found that $a_{T}$, used to construct the master curves of dynamic behavior, well follows the Williams-Landel-Ferry (WLF) function, ${ }^{38} \log a_{T}=-\frac{C_{1} *\left(T-T_{0}\right)}{C_{2}+\left(T-T_{0}\right)}$, where $C_{1}(=58.6)$ and $C_{2}(=504.2)$ are two constants and $T_{0}$ is the reference temperature (= $24{ }^{\circ} \mathrm{C}$ ) (Figure 1c). The $b_{T}$, introduced to account for the small changes in polymer density at different temperatures, is very close to 1, again confirming the thermo-rheologically simple behavior of this gel (Figure 1d).

The dynamic spectrum in Figure 1a shows that over the accessible frequency range, $\omega$, from $10^{-5}$ to $10^{5} \mathrm{rad} / \mathrm{s}$, the PA sample increases its storage and loss moduli gradually with the frequency, and the storage modulus is always higher than the loss modulus. The storage modulus at high frequency is about 100 times of that at low frequency. The mechanical spectrum shows a very broad $\tan \delta$ peak around the frequency of $\omega_{0} \simeq$ $0.3 \mathrm{rad} / \mathrm{s}$, which gives a characteristic relaxation time of $\tau_{0}=1 / \omega_{0} \simeq 3 \mathrm{~s}$ (Figure $1 \mathrm{~b}$ ). These results are in good agreement with our previous report. ${ }^{10}$ The dynamic spectrum indicates that this PA gel has abundant ionic bonds with a characteristic relaxation time around the common time scale of interest, and these bonds are so-called weak bonds in our previous paper. ${ }^{10}$ When $1 / \omega$ is comparable to $\tau_{0}$, most weak bonds break around this time scale, and the hydrogel exhibits the viscoelastic features. When $1 / \omega \gg \tau_{0}$, most weak bonds are relaxed and the gel shows the soft rubbery response. It is noticed that even at a frequency of $10^{-5} \mathrm{rad} / \mathrm{s}$, the sample still exhibits an elastic nature with a 
storage modulus $\left(\sim 10^{5} \mathrm{~Pa}\right)$ higher than the loss modulus, indicating that the longest relaxation time is far longer than $10^{5} \mathrm{~s}$ at $24^{\circ} \mathrm{C}$ (Figure 1a). For $1 / \omega \ll \tau_{0}$, there is no time for most of the weak bonds to break, and the hydrogel responses like in the glassy state with a high dynamic modulus $\left(>2 \times 10^{7} \mathrm{~Pa}\right)$ and a small loss factor $\tan \delta$. So the hydrogel is very stiff and approximately elastic (brittle-like).

Time-temperature equivalence to large strain energy dissipation
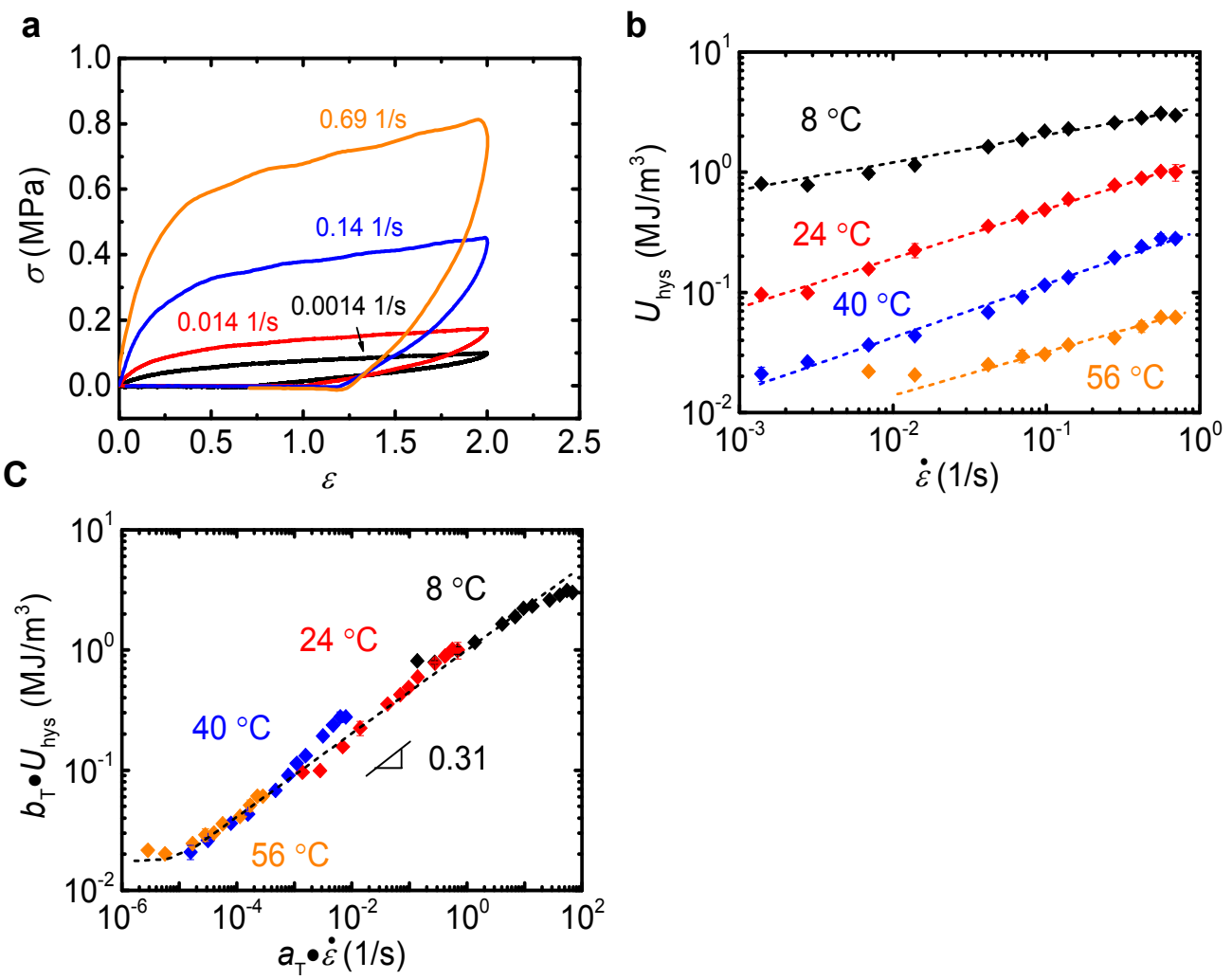

Figure 2 Cyclic tensile behavior of the polyampholyte (PA) hydrogel. a, Cyclic stress - strain curves $(\sigma-\varepsilon)$ at $24{ }^{\circ} \mathrm{C}$ under various cyclic stretch strain rate. b, Relationship between hysteresis $U_{\text {hys }}$ and cyclic strain rate $\dot{\varepsilon}$ under various testing temperature. c, The constructed master curve of hysteresis $U_{h y s}$ against stretch strain 
rate $\dot{\varepsilon}$ using the same shift factors $a_{T}$ and $b_{T}$ determined from the linear rheology in Figure 1c, d, respectively. Reference temperature $T_{0}=24^{\circ} \mathrm{C}$.

For some viscoelastic hydrogels, it has been shown that, the amplitude of strain can be decoupled from the time dependency, resulting in modulus which decreases with the deformation time. ${ }^{41}$ Such decoupling is also approximately applicable for the current system. ${ }^{32}$ This fact suggests that the breakage of weak bonds is dominated by the thermal activation process whereupon the forced debonding effect does not play a substantial role. This motivates us to study the bulk energy dissipation at large strain, and to correlate the data with the small strain behaviors obtained in Figure 1. Figure 2a shows the cyclic tensile behaviors of the sample at a large fixed peak strain $\varepsilon=2$, far above the yielding point. Large hysteresis is observed over the cyclic stress-strain curves, indicating the energy dissipation by the breaking of the weak bonds. It has been confirmed that in the strain range tested, the sample is fully self-resilient and the hysteresis loop disappears after a certain waiting time. ${ }^{10}$ The area of hysteresis markedly increases with increasing cyclic stretch rate, indicating that more ionic bonds contribute to the energy dissipation at high strain rate. As shown in Figure 2b, we found that the relation between the hysteresis energy $U_{\text {hys }}$ and the cyclic strain rate $\dot{\varepsilon}$ follows a power law, $U_{\text {hys }} \sim \dot{\varepsilon}^{m}$ nicely at different temperatures. Raising the temperature expedites the bond breaking, and therefore reduces the hysteresis energy of the hydrogel. The index $m$ varies in the range of $m=0.26 \sim 0.43$ for the temperature performed but does not show a simple trend with temperature.

To investigate the correlation between the hysteresis data and the linear rheological data, we use the same shift factors $a_{T}$ and $b_{T}$ obtained from the linear rheology in Figure 
1c and Figure 1d to build a master curve of energy dissipation. We found that the hysteresis energy at the large strain can be reduced to a master curve with the strain rate, as shown in Figure 2c. This result means that, the effect of temperature on the hysteresis can be accounted for simply by applying $a_{T}$ and $b_{T}$ to $\dot{\varepsilon}$ at a reference temperature $\left(T_{0}=24^{\circ} \mathrm{C}\right)$

$U_{h y s}(\dot{\varepsilon}, \mathrm{T})=\mathrm{b}_{\mathrm{T}} \mathrm{U}_{\text {hys }}\left(\mathrm{a}_{\mathrm{T}} \dot{\varepsilon}, \mathrm{T}_{0}\right)$

This result demonstrates that hysteresis energy also follows the time-temperature superposition principle with the same shift factors as those for the small strain rheological observation. This means that the linear rheology is also applicable to the bulk energy dissipation at large deformation, and the bond breaking process is thermally activated, and hardly depends on the applied strain. This is consistent with the strain-amplitude-time decoupling observed in tensile tests of the material. ${ }^{32}$ In the range of strain rate $10^{-5} \sim 10^{2} \mathrm{~s}^{-1}$, a scaling relationship is found as, $U_{\text {hys }} \sim \dot{\varepsilon}^{0.31}$.

Time-temperature equivalence to tearing energy 
a

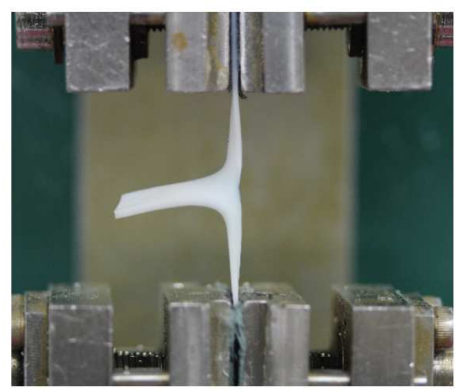

C

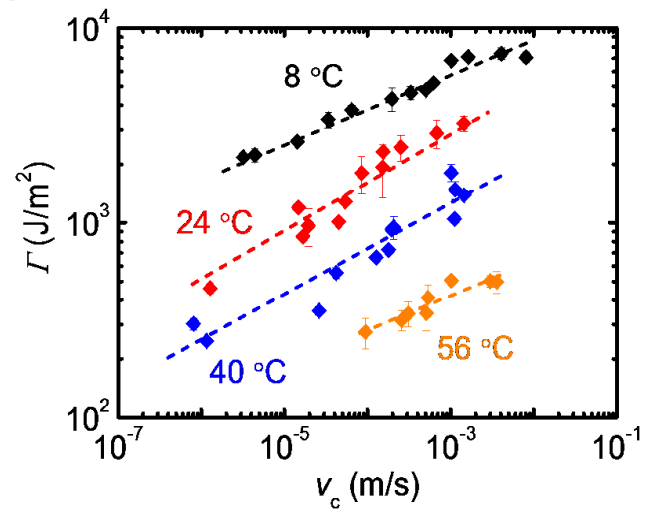

b

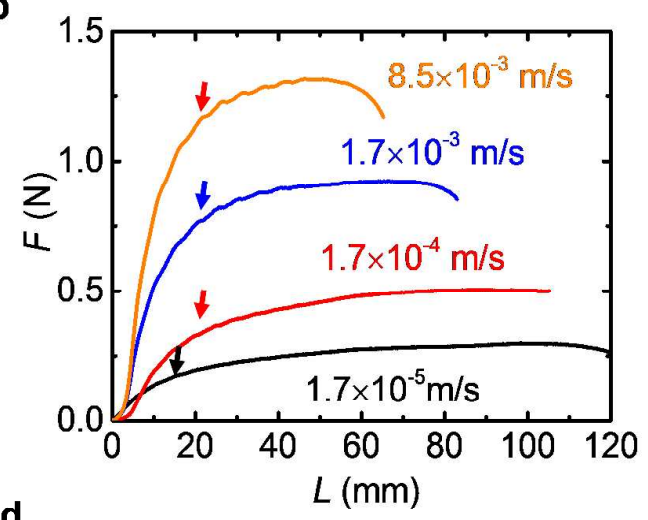

d

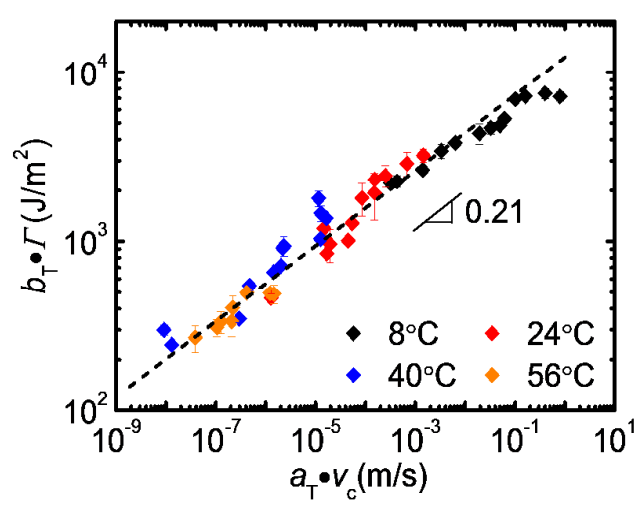

Figure 3 Fracture behavior of the polyampholyte (PA) hydrogel. a, An image of the sample during a tearing test. b, Force - displacement curves $(F-L)$ of the tearing test at $24{ }^{\circ} \mathrm{C}$ at different tearing velocities. c, Relationship between tearing energy $\Gamma$ and crack velocity $V_{c}$ at different testing temperature. d, The constructed master curves of tearing energy $\Gamma$ against crack velocity $V_{c}$ using the same shift factors $a_{T}$ and $b_{T}$ determined from the linear rheology in Figure 1c and Figure 1d, respectively. Reference temperature $T_{0}=24{ }^{\circ} \mathrm{C}$. The arrows in the inserts of Figure $2 \mathrm{~b}$ correspond to the positions that crack propagation starts.

The tearing energy, $\Gamma$, namely the energy required to propagate a crack by a unit area, was measured by the tearing test, as shown in Figure 3a. The typical force-displacement curves $(F-L)$ obtained from the tearing test with different tearing velocities, $V_{t}$, at 24 
${ }^{\circ} \mathrm{C}$ are presented in Figure $3 \mathrm{~b}$. At a given tearing velocity, the two legs of notched sample are elongated without advancing of the crack tip and the force increases with elongation until reaching a plateau, then the crack propagation starts at the position shown by the arrows in the figure and soon reaches a constant velocity. With the increase of the tearing velocity, the force required to elongate the sample and to advance the crack increases, as the bond life time becomes relatively longer for a shorter observation time. To directly relate the tearing energy to local crack propagation process, the constant crack velocity, $V_{c}$, was estimated from a synchronized video. Except for the points at high tearing velocity, all the observed crack velocities at various temperatures are slightly lower than the applied tearing velocities over a wide range, as shown in Figure S2. We correlate the calculated tearing energy to the crack velocity under various temperatures, and the results are shown in Figure 3c. Raising the temperature expedites bond breaking, and therefore reduces the tearing energy of the gel. Tearing energy increases with crack velocity, following power law relations $\Gamma \sim \mathrm{V}_{\mathrm{c}}^{\alpha}$ nicely at different temperatures. The index of the power law, $\alpha$, varies in the range of $0.18 \sim 0.28$ for the temperature performed but does not show a simple trend with temperature.

It has been shown that, for viscoelastic rubbers, the energy required to advance a fracture plane by a unit area includes the intrinsic fracture energy of the polymer network ahead of the crack tip and the bulk viscoelastic energy dissipated around the crack tip. ${ }^{19-28}$ The tearing energy of rubber strongly depends on the crack velocity, and is greatly enhanced by viscous dissipative losses that are several orders of magnitude larger than the intrinsic fracture energy of the polymer network. ${ }^{23-25,27,28}$ The fracture energy of viscoelastic solids is usually described by the following equation: $\Gamma\left(V_{c}, \mathrm{~T}\right)=$ $\Gamma_{0}\left(1+\mathrm{f}\left(V_{c}, \mathrm{~T}\right)\right)$, where the viscoelastic dissipation term $\mathrm{f}\left(V_{c}, \mathrm{~T}\right)$ is related to crack 
velocity $V_{c}$ and temperature $T$, and $\Gamma_{0}$ is a threshold value of the polymer network below which no fracture occurs..$^{25,27,28,42,43}$ During crack propagation, the intrinsic fracture energy of a polymer network involves several complex processes, including chain breaking, cavitation formation, and so on, while the bulk viscoelastic energy dissipation only involves the viscoelastic deformation process around the crack tip. When the fracture energy is dominated by the viscoelastic energy dissipation, the fracture behavior is governed by the bulk viscoelasticity and the fracture properties may be correlated to viscoelastic data (Figure 2c). That is, the effect of temperature on the fracture energy can be accounted for simply by applying shift factor $a_{T}$ obtained from rheological test to the crack velocity $V_{c}, f\left(V_{c}, T\right)=f\left(a_{T} V_{c}, T_{0}\right)$. Therefore, the tearing energy obtained for a higher velocity at a given temperature has the same value as that measured at the lower velocity and low temperature according to the time-temperature equivalence. $^{27,28}$

Following this equivalence, we found that, as shown in Figure 3d, all the tearing energy data measured at different temperatures and crack velocities can be reduced to a nice master curve at a reference temperature $\left(T_{0}=24{ }^{\circ} \mathrm{C}\right)$ when the the same shift factors $a_{T}$ and $b_{T}$ determined from the linear dynamic measurement in Figure 1c and Figure 1d are used, respectively. The result indicates that the equivalence holds for the PA hydrogel: $\Gamma\left(V_{c}, T\right)=b_{T} \Gamma\left(a_{T} V_{c}, T_{0}\right)$. Therefore, the tearing energy is dominantly dissipated by the bulk viscoelasticity around the crack tip, governed by the thermally activated bond dissociation processes. In the observed crack velocity range $\left(10^{-8} \mathrm{~m} / \mathrm{s} \sim\right.$ $10^{0} \mathrm{~m} / \mathrm{s}$ ), the relationship between the tearing energy and crack velocity follows a power law, $\Gamma \sim \mathrm{V}_{\mathrm{c}}^{\alpha}$ with index $\alpha=0.21$. This index value is slightly lower than that of the 
power law relation between the hysteresis energy and stretching strain rate, $U_{\text {hys }} \sim \dot{\varepsilon}^{0.31}$ (Figure 2c).

It is noticed that even at a crack velocity as low as $10^{-8} \mathrm{~m} / \mathrm{s}$ (Figure $3 \mathrm{~d}$ ), the tearing energy still shows a velocity dependence. Furthermore, the tearing energy is $\sim 200 \mathrm{~J} / \mathrm{m}^{2}$ at such low velocity. This tearing energy, regardless of the $50 w t \%$ water content of the PA gel, is much larger than the usual threshold value of rubber materials $\left(\sim 50 \mathrm{~J} / \mathrm{m}^{2}\right)$ that do not contain any solvents. ${ }^{44}$ This indicates that even at such a low crack velocity, the bulk energy dissipation still dominates the tearing energy in PA gel. This is in consistent with the extremely long creep time of the system. ${ }^{31}$

\section{Correlation between relaxation modulus and fracture energy}
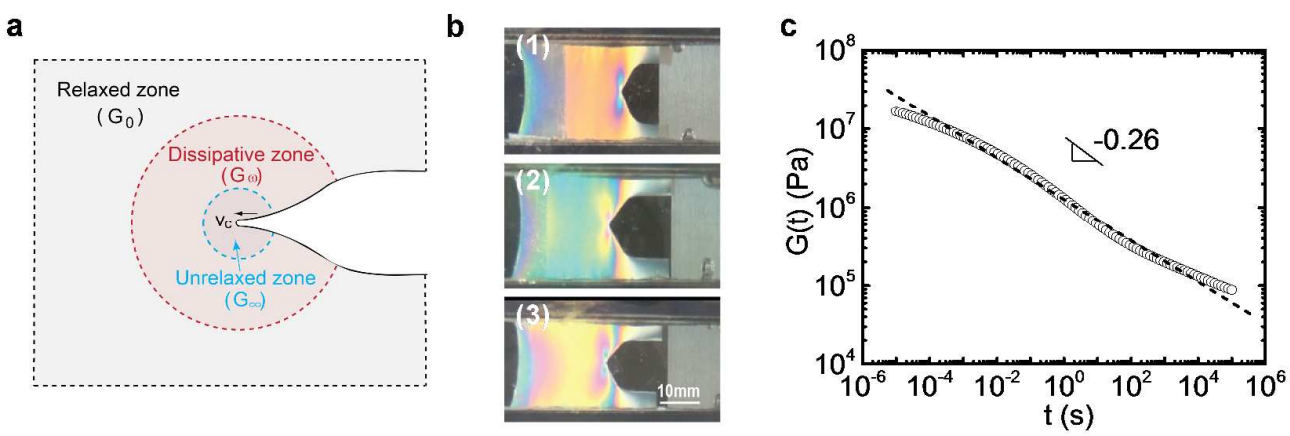

Figure 4 (a) The energy dissipative model for the fracture of viscoelastic solids. The energy dissipation profile at the crack tip consists of three different zones depending on the distance $r$ from the crack tip: the unrelaxed zone at the crack tip $\left(G_{\infty}\right)$, viscoelastic energy dissipative zone around the crack tip $\left(G_{\omega}\right)$, and the fully relaxed zone that is far from the crack tip $\left(G_{0}\right) \cdot{ }^{21,25,27,28}$ (b) Circular birefringence images of the PA hydrogel undergoing crack growth at velocities, $1.7 \times 10^{-5} \mathrm{~m} / \mathrm{s}(1), 1.7 \times 10^{-4} \mathrm{~m} / \mathrm{s}(2)$, and $1.7 \times$ $10^{-3} \mathrm{~m} / \mathrm{s}$ (3) during pure shear test. The images were taken at the strain $\varepsilon=1.5$. The 
data are adopted from Ref 32. (c) The relaxation modulus, $G(\mathrm{t})$, obtained from the dynamic modulus, $G(\omega)$, at various angular frequencies in Figure 1(a).

Here, we intend to correlate the dynamic mechanical spectrum with the crack velocity dependence of the fracture energy. For this purpose, let us first revisit the bulk energy dissipative model for the fracture of viscoelastic solid materials. ${ }^{21,25,27,28}$ The model assumes that the viscoelastic solid is described by a single relaxation time $\tau$, with two plateau moduli, $G_{0}$ and $G_{\infty}$, at low frequency $(\omega \tau \ll 1)$ and high frequency $(\omega \tau \gg 1)$, respectively. Given that the deformation rate at a distance $r$ from the crack tip is characterized by the angular frequency $\omega=V_{c} / r$, the energy dissipation profile at the crack tip consists of three different zones depending on the distance $r$ from the crack tip, (Figure 4a). ${ }^{21,25,27,28}$ Near the crack tip, where $r$ is very small, the zone is in the hard glassy state with modulus $G_{\infty}$ as frequency $\omega$ becomes very high $(\omega \tau \gg 1)$. Far from the crack tip with large $r(\omega \tau \ll 1)$, the zone is in the rubbery state with modulus $G_{0}$. At the intermediate distance $r(\omega \tau \sim 1)$, the zone is in the viscoelastic state, giving large energy dissipation. In our previous work on the PA gels, we indeed observed the above-described regions by pure shear test under circular polarized light (Figure 4b). ${ }^{32}$ Given a crack velocity $V_{c} \sim 1.7 \times 10^{-5} \mathrm{~m} / \mathrm{s}$ at strain $\varepsilon=1.5$ (Figure $4 b(1))$, the region near the crack tip was very stiff, and the crack advanced with a constant opening angle. Ahead of the crack tip, the region had a complicated stress field, and there was a large yielding zone with significant viscoelastic dissipation, as indicated by the butterfly-shaped birefringence pattern. The region far away from the crack tip corresponded to the pure shear zone with a uniform birefringence color. With increasing crack advancing velocity, the crack opening angle decreased (Figure $4 b(2)$, (3)), 
indicating that the region near the crack tip became more rigid. The size of the yielding zone, where most viscoelastic dissipation took place, increased with the crack velocity (Figure $4 b$ ). In the frame work of the bulk energy dissipation model, several theoretical works have discussed the crack velocity dependence of fracture energy and its correlation with the viscoelastic relaxation spectrum $G_{\omega}$ in the transition region. ${ }^{19-22,25-}$ ${ }^{28}$ Several theories have tried to correlate the power-law relationship of fracture energy $\Gamma$ and crack velocity $V_{c}, \Gamma \sim \mathrm{V}_{\mathrm{c}}^{\alpha}$, to the linear viscoelastic response of bulk materials, using the creep compliance function by Schapery or stress relaxation function by Persson. ${ }^{19,27,28}$ For example, the theory predicts that the exponent $\alpha$ of $\Gamma \sim \mathrm{V}_{\mathrm{c}}^{\alpha}$ and the exponent $\kappa$ of $G(\mathrm{t}) \sim t^{-\kappa}$, the power law relation of the relaxation modulus $G(\mathrm{t})$ and time $t$ in the viscoelastic transition region, can be correlated as follows:

$$
\alpha=\kappa /(1+\kappa)
$$

This theoretical relation has been verified by several experiments on rubbers. ${ }^{45,46}$ Here, we analyze our results using Persson's theory. Before doing this, we should check whether the frequency range over which the viscoelastic spectrum was measured (Figure 1a, $a_{T} \omega=10^{-5} \sim 10^{5} \mathrm{rad} / \mathrm{s}$ ) covers the deformation frequency range in front of the crack tip during crack propagation. Given the lower limit of $r$ the size of monomer $(\sim 0.3 \mathrm{~nm})$ and the upper limit of the width of the sample arm $(\sim 3.8 \mathrm{~mm})$, we can estimate the deformation frequency range using the relation $\omega=V_{c} / r$ (Figure 3d). For $V_{c}=10^{-8} \mathrm{~m} / \mathrm{s} \sim 1 \mathrm{~m} / \mathrm{s}$, the high angular frequency bound close to the crack tip is in the range $\omega=3 \times 10^{-1} \sim 3 \times 10^{9} \mathrm{rad} / \mathrm{s}$ using $r=0.3 \mathrm{~nm}$. Although our observed high frequency bound $\left(\sim 10^{5} \mathrm{rad} / \mathrm{s}\right)$ is much lower than $3 \times 10^{9} \mathrm{rad} / \mathrm{s}$, and can only cover the range $r>10 \mu \mathrm{m}$ for the high velocity $V_{c}=1 \mathrm{~m} / \mathrm{s}$, the energy dissipation at the zone $r$ $<10 \mu \mathrm{m}$ may not make dominant contribution to the tearing energy for two reasons: 1) 
the $\tan \delta$ peak is around $\omega_{0}=0.3 \mathrm{rad} / \mathrm{s}$ (Figure $1 \mathrm{~b}$ ), which corresponds to $r=0.03 \mu \mathrm{m}$ $\sim 3.3 \mathrm{~m}$ for the measured crack velocity range of $V_{c}=10^{-8} \mathrm{~m} / \mathrm{s} \sim 1 \mathrm{~m} / \mathrm{s}$. This means, except at the low crack velocity limit, the viscoelastic region is located at a distance much larger than $r=10 \mu \mathrm{m} ; 2)$ as the dynamic properties only depend on the distance to the crack tip $r$, the energy dissipation in a zone with distance $r$ and width $d r$ is proportional to $\sim 2 \pi r d r$. Accordingly, even at a relatively small crack velocity, the region close to the crack tip contributes less to the energy dissipation than that of the large $r$. This implies that bulk viscoelastic energy dissipation dominates the total fracture energy of samples under the observation time range. This is why the measured fracture energy $\left(\sim 200 \mathrm{~J} / \mathrm{m}^{2}\right)$ at the minimum crack velocity $\sim 10^{-8} \mathrm{~m} / \mathrm{s}$ is larger than the common value of the intrinsic fracture energy of polymer networks (Figure 3d).

To analyze our results using Persson's theory, we transform the data of dynamic modulus $G^{\prime}(\omega)$ and $G^{\prime \prime}(\omega)$ in Figure 1a into the relaxation modulus $G(\mathrm{t})$ based on the linear viscoelasticity theory through the intermediate relaxation time spectrum $H(\tau) .{ }^{47,48}$ Both the relaxation modulus and dynamic modulus can be calculated by superimposing Maxwell relaxation modes, and each relaxation mode has a characteristic relaxation time $\tau$ :

$\mathrm{G}(\mathrm{t})=\mathrm{G}_{\mathrm{e}}+\int_{-\infty}^{+\infty} \mathrm{H}(\ln \tau) \mathrm{e}^{-\mathrm{t} / \tau} \mathrm{dln} \tau$

$G^{\prime}(\omega)=\int_{-\infty}^{+\infty} H(\ln \tau) \frac{\omega^{2} \tau^{2}}{1+\omega^{2} \tau^{2}} d \ln \tau$

$G^{\prime \prime}(\omega)=\int_{-\infty}^{+\infty} H(\ln \tau) \frac{\omega \tau}{1+\omega^{2} \tau^{2}} d \ln \tau$

Here, $G_{\mathrm{e}}$ is the equilibrium modulus which is zero for physical PA gels. The continuous relaxation time spectrum $H(\tau)$, as shown in Figure S3, is calculated from the dynamic modulus $G^{\prime}(\omega)$ and $G^{\prime \prime}(\omega)$ by using the build-in non-linear regression 
procedures of the ARES rheometer. ${ }^{47,48}$ Then, $G(\mathrm{t})$ is obtained from $H(\tau)$, as shown in Figure 4c. By fitting the data with the power law relation, $G(\mathrm{t}) \sim t^{-\kappa}$ in the range of $10^{-5}$ to $10^{5} \mathrm{~s}$, we obtained the exponent $\kappa=0.26$. Substituting the $\kappa$ value into Equation 4, one can predict that the exponent of the power law of the tearing energy and crack velocity, $\alpha$, is $0.21, \Gamma \sim \mathrm{V}_{\mathrm{c}}^{0.21}$. This value obtained using the linear viscoelastic fracture theory is in excellent agreement with the experimental observation $\left(\Gamma \sim \mathrm{V}_{\mathrm{c}}^{0.21}\right)$ (Figure 3d). The above results show that, at this observation time scale, the crack propagation energy indeed has the form $\Gamma\left(V_{\mathrm{c}}, T\right)=\Gamma_{0}\left(1+\mathrm{f}\left(V_{\mathrm{c}}, T\right)\right)$, and is dominated by the viscoelastic energy term $\mathrm{f}\left(V_{\mathrm{c}}, T\right)$. The viscoelastic theory is able to predict the fracture data from the linear viscoelastic spectrum.

When comparing the experimental results with the theoretically predicted value, two remarks should be made. One is that a single relaxation mode at this range of stress relaxation function is considered in Figure 4c. Several relaxation modes can be observed from the curve of $G(\mathrm{t}) \sim t^{-\kappa}$ in Figure 4c, which leads to over simplification of the theoretical consideration. Another is the applicability of linear fracture mechanics in the region close to the crack tip. In the region that is far away from the crack tip, the self-healing behavior of the gel makes the breaking and healing of physical bonds independent of strain and strain rate at small values, and the rupture of bonds can be reformed again with a shorter healing time than that of breaking time. ${ }^{15,32,49}$ The healed bonds do not carry load and cannot be stretched to large deformation. The fracture energy is dissipated by rupture of bonds and releasing the elastic energy of the chain. The applied linear fracture theory to this region is suitable. However, close to the crack tip the local deformation and deformation rates become very high, and polymer chains can be stretched to a very high level before breaking of the physical bonds (strain 
hardening). In such case the decoupling of amplitude of strain from the time dependency fails to hold for the current system and the forced debonding effect may play a role, where the linear fracture mechanics may no longer apply, ${ }^{32,49}$ although the contribution to the fracture energy in this range is very low. How the local strain fields around the crack tip effect crack propagation behavior should be considered in future work.

\section{CONCLUSIONS}

Although the physical polyempholyte hydrogels show a phase separated structure and a very broad relaxation spectrum, the dynamic modulus, the tensile hysteresis, and the tearing energy all follow the principle of time-temperature superposition. The tearing energy increases with observed crack velocity, as a power law relationship, $\Gamma \sim V_{c}^{0.21}$ and is correlated to the relaxation modulus $G(\mathrm{t}) \sim t^{-0.26}$ by Persson's viscoelastic theory. These results indicate that 1) The physical PA gel exhibits the thermorheologically simple behaviour and all the ionic bonds, either strong or weak, have the same temperature dependence; 2) The tearing energy of the PA hydrogel is dominated by the bulk viscoelastic energy dissipation in front of the crack tip; 3) Linear viscoelastic theory is applicable for this supramolecular hydrogel system containing solvent, as like solid rubbers. The simple correlation between the dynamic spectrum of fracture energy and the relaxation modulus indicates that the dynamic mechanical spectrum can be used as a fingerprint for designing tough viscoelastic hydrogels.

\section{ASSOCIATED CONTENT}

\section{Supporting Information}

The Supporting Information is available free of charge on the ACS Publications website. Figure S1 Linear dynamic behavior of physical polyampholyte hydrogels; Figure S2 
Relationship between the crack velocity $V_{c}$ and tearing velocity $V_{t}$ at different temperatures; Figure S3 The relaxation time spectrum of the PA gel calculated from the data of dynamic modulus.

\section{AUTHOR INFORMATION}

Corresponding Authors

*(T. L. S) E-mail: suntl@sci.hokudai.ac.jp

*(J. P. G) E-mail: gong@sci.hokudai.ac.jp

Tel \& FAX: +81-(0)11-706- 9011

\section{NOTES}

The authors declare no competing financial interest.

\section{ACKNOWLEDGEMENTS}

This research was financially supported by Grant-in-Aid for Scientific Research (S) (No. 124225006) from the Japan Society for the Promotion of Science (JSPS). This research was also partially funded by ImPACT Program of Council for Science, Technology and Innovation (Cabinet Office, Government of Japan).

\section{REFERENCES}

(1) Drury, J. L.; Mooney, D. J. Hydrogels for Tissue Engineering: Scaffold Design Variables and Applications. Biomaterials 2003, 24 (24), 4337-4351.

(2) Gong, J. P. Why are Double Network Hydrogels So Tough? Soft Matter 2010, 6 (12), 2583-2590.

(3) Gong, J. P.; Katsuyama, Y.; Kurokawa, T.; Osada, Y. Double-Network Hydrogels with Extremely High Mechanical Strength. Adv. Mater. 2003, 15 (14), $1155-1158$. 
(4) Guo, M.; Pitet, L. M.; Wyss, H. M.; Vos, M.; Dankers, P. Y. W.; Meijer, E. W. Tough Stimuli-Responsive Supramolecular Hydrogels with Hydrogen-Bonding Network Junctions. J. Am. Chem. Soc. 2014, 136 (19), 6969-6977.

Zhang, H. J.; Sun, T. L.; Zhang, A. K.; Ikura, Y.; Nakajima, T.; Nonoyama, T.; Kurokawa, T.; Ito, O.; Ishitobi, H.; Gong, J. P. Tough Physical Double-Network Hydrogels Based on Amphiphilic Triblock Copolymers. Adv. Mater. 2016, 28 (24), 4884-4890.

(6) Luo, F.; Sun, T. L.; Nakajima, T.; Kurokawa, T.; Zhao, Y.; Sato, K.; Ihsan, A. B.; Li, X.; Guo, H.; Gong, J. P. Oppositely Charged Polyelectrolytes Form Tough, Self-healing, and Rebuildable Hydrogels. Adv. Mater. 2015, 27 (17), 2722-2727.

(7) Moutos, F. T.; Freed, L. E.; Guilak, F. A Biomimetic Three-Dimensional Woven Composite Scaffold for Functional Tissue Engineering of Cartilage. Nat. Mater. 2007, $6(2), 162-167$.

(8) Naficy, S.; Brown, H. R.; Razal, J. M.; Spinks, G. M.; Whitten, P. G. Progress Toward Robust Polymer Hydrogels. Aust. J. Chem. 2011, 64 (8), 1007-1025.

(9) Sun, J.-Y.; Zhao, X.; Illeperuma, W. R. K.; Chaudhuri, O.; Oh, K. H.; Mooney, D. J.; Vlassak, J. J.; Suo, Z. Highly Stretchable and Tough hydrogels. Nature 2012, 489 (7414), 133-136.

(10) Sun, T. L.; Kurokawa, T.; Kuroda, S.; Ihsan, A. B.; Akasaki, T.; Sato, K.; Haque, M. A.; Nakajima, T.; Gong, J. P. Physical Hydrogels Composed of Polyampholytes Demonstrate High Toughness and Viscoelasticity. Nat. Mater. 2013, 12 (10), 932-937.

(11) Huang, T.; Xu, H. G.; Jiao, K. X.; Zhu, L. P.; Brown, H. R.; Wang, H. L. A Novel Hydrogel with High Mechanical Strength: A Macromolecular Microsphere 
Composite Hydrogel. Adv. Mater. 2007, 19 (12), 1622-1626.

(12) Haraguchi, K.; Takehisa, T. Nanocomposite Hydrogels: A Unique Organic-Inorganic Network Structure with Extraordinary Mechanical, Optical, and Swelling/De-swelling Properties. Adv. Mater. 2002, 14 (16), 1120-1124.

(13) Sun, T. L.; Luo, F.; Kurokawa, T.; Karobi, S. N.; Nakajima, T.; Gong, J. P. Molecular Structure of Self-Healing Polyampholyte Hydrogels Analyzed from Tensile Behaviors. Soft Matter 2015, 11 (48), 9355-9366.

(14) Rose, S.; Dizeux, A.; Narita, T.; Hourdet, D.; Marcellan, A. Time Dependence of Dissipative and Recovery Processes in Nanohybrid Hydrogels. Macromolecules 2013, 46 (10), 4095-4104.

(15) Long, R.; Mayumi, K.; Creton, C.; Narita, T.; Hui, C. -Y. Time Dependent Behavior of a Dual Cross-Link Self-Healing Gel: Theory and Experiments. Macromolecules 2014, 47 (20), 7243-7250.

(16) Na, Y. -H.; Tanaka, Y.; Kawauchi, Y.; Furukawa, H.; Sumiyoshi, T.; Gong, J. P.; Osada, Y. Necking Phenomenon of Double-Network Gels. Macromolecules 2006, 39 (14), 4641-4645.

(17) Yu, Q. M.; Tanaka, Y.; Furukawa, H.; Kurokawa, T.; Gong, J. P. Direct Observation of Damage Zone around Crack Tips in Double-Network Gels. Macromolecules 2009, 42 (12), 3852-3855.

(18) Ducrot, E.; Chen, Y.; Bulters, M.; Sijbesma, R. P.; Creton, C. Toughening Elastomers with Sacrificial Bonds and Watching Them Break. Science 2014, 344 (6180), 186-189.

(19) Schapery, R. A. A Theory of Crack Initiation and Growth in Viscoelastic Media II. Approximate Methods of Analysis. Int. J. Fract. 1975, 11 (3), 369-388. 
(20) Greenwood, J. A.; Johnson, K. L. The Mechanics of Adhesion of Viscoelastic Solids. Philos. Mag. A 1981, 43 (3), 697-711.

(21) Hui, C. -Y.; Xu, D. -B; Kramer, E. J. A Fracture Model for a Weak Interface in a Viscoelastic Material (Small Scale Yielding Analysis). J. Appl. Phys. 1992, 72 (8), $3294-3304$.

(22) Bowen, J. M.; Knauss, W. G. The Characterization of the Energy of Fracture at or Near Interfaces Between Viscoelastic Solids. J. Adhes. 1992, 39 (1), 43-59.

(23) Gent, A. N.; Lai, S. -M. Interfacial Bonding, Energy Dissipation, and Adhesion. J. Polym. Sci. Part B Polym. Phys. 1994, 32 (8), 1543-1555.

(24) Gent, A. N. Adhesion and Strength of Viscoelastic Solids. Is There a Relationship between Adhesion and Bulk Properties? Langmuir 1996, 12 (19), $4492-4496$.

(25) de Gennes, P. G. Soft Adhesives. Langmuir 1996, 12 (19), 4497-4500.

(26) Saulnier, F.; Ondarcuhu, T.; Aradian, A.; Raphael, E. Adhesion between a Viscoelastic Material and a Solid Surface. Macromolecules 2004, 37 (3), 10671075.

(27) Persson, B. N. J.; Brener, E. A. Crack Propagation in Viscoelastic Solids. Phys. Rev. E 2005, 71 (3), 036123.

(28) Persson, B. N. J.; Albohr, O.; Heinrich, G.; Ueba, H. Crack Propagation in Rubber-Like Materials. J. Phys.: Condens. Matter 2005, 17 (44), R1071-R1142.

(29) Ihsan, A. B.; Sun, T. L.; Kuroda, S.; Haque, M. A.; Kurokawa, T.; Nakajima, T.; Gong, J. P. A Phase Diagram of Neutral Polyampholyte - from Solution to Tough Hydrogel. J. Mater. Chem. B 2013, 1 (36), 4555-4562.

(30) Ihsan, A. B.; Sun, T. L.; Kurokawa, T.; Karobi, S. N.; Nakajima, T.; Nonoyama, 
T.; Roy, C. K.; Luo, F.; Gong, J. P. Self-Healing Behaviors of Tough

Polyampholyte Hydrogels. Macromolecules 2016, 49 (11), 4245-4252.

(31) Karobi, S. N.; Sun, T. L.; Kurokawa, T.; Luo, F.; Nakajima, T.; Nonoyama, T.;

Gong, J. P. Creep Behavior and Delayed Fracture of Tough Polyampholyte

Hydrogels by Tensile Test. Macromolecules 2016, 49 (15), 5630-5636.

(32) Luo, F.; Sun, T. L.; Nakajima, T.; Kurokawa, T.; Zhao, Y.; Ihsan, A. B.; Guo, H. L.; Li, X. F.; Gong, J. P. Crack Blunting and Advancing Behaviors of Tough and Self-Healing Polyampholyte Hydrogel. Macromolecules 2014, 47 (17), 60376046.

(33) Cui, K.; Sun, T. L.; Kurokawa, T.; Nakajima, T.; Nonoyama, T.; Chen, L.; Gong, J. P. Stretching-Induced Ion Complexation in Physical Polyampholyte Hydrogels. Soft Matter 2016, 12 (43), 8833-8840.

(34) Sato, K.; Nakajima, T.; Hisamatsu, T.; Nonoyama, T.; Kurokawa, T.; Gong, J. P. Phase-Separation-Induced Anomalous Stiffening, Toughening, and Self-Healing of Polyacrylamide Gels. Adv. Mater. 2015, 27 (43), 6990-6998.

(35) Guo, H.; Sanson, N.; Hourdet, D.; Marcellan, A. Thermoresponsive Toughening with Crack Bifurcation in Phase-Separated Hydrogels under Isochoric Conditions. Adv. Mater. 2016, 28 (28), 5857-5864.

(36) Cui, K.; Sun, T. L.; Kurokawa, T.; Nakajima, T.; Nonoyama, T.; Chen, L.; Gong, J. P. to be submitted to Macromolecules.

(37) Long, R.; Hui, C.-Y. Fracture Toughness of Hydrogels: Measurement and Interpretation. Soft Matter 2016, 12 (39), 8069-8086.

(38) Rubinstein, M.; Colby, R. H. Polymer Physics; Oxford University Press Inc.: New York, 2003. 
(39) Plazek, D. J. 1995 Bingham Medal Address: Oh, Thermorheological Simplicity, Wherefore Art Thou? J. Rheol. 1996, 40 (6), 987-1014.

(40) van Gurp, M.; Palmen, J. Time-Temperature Superposition for Polymeric Blends. J. Rheol. Bull. 1998, 67, 5-8.

(41) Mayumi, K.; Marcellan, A.; Ducouret, G.; Creton, C.; Narita, T. Stress-Strain Relationship of Highly Stretchable Dual Cross-Link Gels: Separability of Strain and Time Effect. ACS Macro Lett. 2013, 2 (12), 1065-1068.

(42) Cristiano, A.; Marcellan, A.; Keestra, B. J.; Steeman, P.; Creton, C. Fracture of Model Polyurethane Elastomeric Networks. J. Polym. Sci. Part B Polym. Phys. 2011, 49 (5), 355-367.

(43) Mzabi, S.; Berghezan, D.; Roux, S.; Hild, F.; Creton, C. A Critical Local Energy Release Rate Criterion for Fatigue Fracture of Elastomers. J. Polym. Sci. Part B Polym. Phys. 2011, 49 (21), 1518-1524.

(44) Lake, G. J.; Thomas, A. G. The Strength of Highly Elastic Materials. Proc. $R$. Soc. A Math. Phys. Eng. Sci. 1967, 300 (1460), 108-119.

(45) Klüppel, M. Evaluation of Viscoelastic Master Curves of Filled Elastomers and Applications to Fracture Mechanics. J. Phys.: Condens. Matter 2009, 21 (3), 035104.

(46) Morishita, Y.; Tsunoda, K.; Urayama, K. Velocity Transition in the Crack Growth Dynamics of Filled Elastomers: Contributions of Nonlinear Viscoelasticity. Phys. Rev. E 2016, 93 (4), 043001.

(47) Honerkamp, J.; Weese, J. A Nonlinear Regularization Method for the Calculation of Relaxation Spectra. Rheol. Acta 1993, 32 (1), 65-73. 
(48) Elster, C.; Honerkamp, J.; Weese, J. Using Regularization Methods for the Determination of Relaxation and Retardation Spectra of Polymeric Liquids. Rheol. Acta 1992,31 (2), 161-174.

(49) Mayumi, K.; Guo, J.; Narita, T.; Hui, C. -Y.; Creton, C. Fracture of Dual Crosslink Gels with Permanent and Transient Crosslinks. Extrem. Mech. Lett. 2016, 6, 52-59. 


\section{Table of Contents Graphic Bulk Energy Dissipation Mechanism for the Fracture of Tough and Self-Healing Hydrogels}

Tao Lin Sun ${ }^{1,2 *}$, Feng Luo ${ }^{1}$, Wei Hong ${ }^{2,3}$, Kunpeng Cui ${ }^{1}$, Yiwan Huang ${ }^{4}$, Hui Jie Zhang $^{4}$, Daniel R. King ${ }^{1,2}$, Takayuki Kurokawa ${ }^{1,2}$, Tasuku Nakajima ${ }^{1,2}$, Jian Ping Gong $^{1,2 *}$

${ }^{1}$ Laboratory of Soft and Wet Matter, Faculty of Advanced Life Science, Hokkaido

University, Sapporo 001-0021, Japan

${ }^{2}$ Global Station for Soft Matter, Global Institution for Collaborative Research and Education (GI-CoRE), Hokkaido University

${ }^{3}$ Department of Aerospace Engineering, Iowa State University of Science and Technology, Ames, IA 50010, USA

${ }^{4}$ Laboratory of Soft and Wet Matter, Graduate School of Life Science, Hokkaido

University, Sapporo 001-0021, Japan

Corresponding Authors

*(T. L. S): E-mail: suntl@sci.hokudai.ac.jp

*(J. P. G): E-mail: gong@s, sci.hokudai.ac.jp

Tel \& FAX: +81-(0)11-706- 9011

Key Words: Polyampholyte hydrogel; Viscoelasticity; Fracture behavior; Self-healing; Ionic bond; Time-temperature superposition 

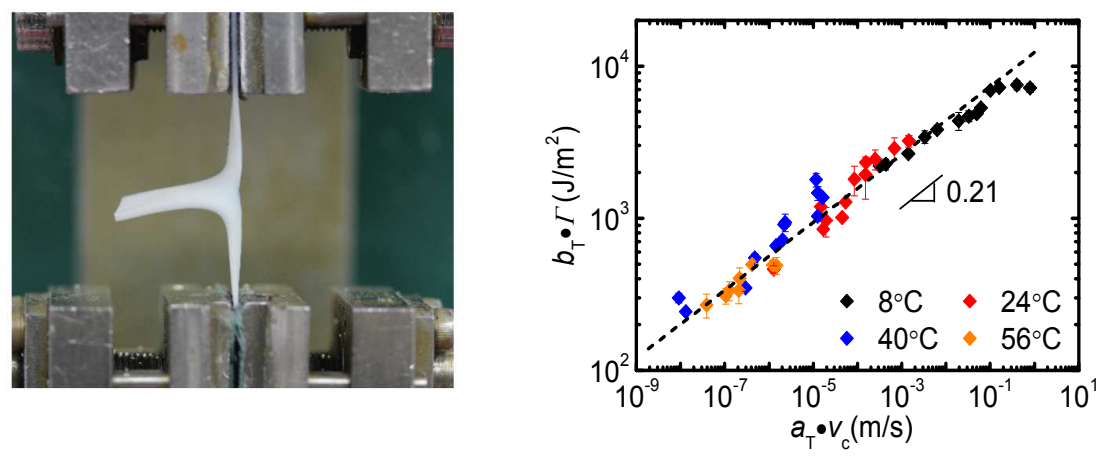

1

2

3

4

5

7

8

9

10

11

12

13

14

16

17

18

19

20

21

22

23

24

25

26

27

28

29

30

31

32

33

34

35

36

37

38

39

40

41

42

43

44

45

46

47

48

49

50

51

52

53

54

55

56

57

58

59 